\title{
Selective localization of $\lg G$ from cerebrospinal fluid to brain parenchyma
}

\author{
Marlene Thorsen Mørch', Sofie Forsberg Sørensen ${ }^{1}$, Reza Khorooshi', Nasrin Asgari ${ }^{1,2}$ and Trevor Owens ${ }^{1 *}$ (D)
}

\begin{abstract}
Background: Encounter of autoantibodies with specific antigens can lead to hypersensitivity reactions and pathology. In multiple sclerosis and neuromyelitis optica spectrum disease (NMOSD), immunoglobulin-G (IgG) deposition has been observed in pathological lesions in the central nervous system. The paradigmatic autoantibodies in NMOSD are specific for the water channel aquaporin-4, localized to astrocytic end-feet at the blood-brain barrier and ependymal cells at the cerebrospinal fluid-brain barrier. We have previously observed that lgG antibodies from NMO patients (NMO-lgG) access brain parenchyma from the cerebrospinal fluid and induce subpial and periventricular NMO-like lesions and blood-brain barrier breakdown, in a complement-dependent manner.
\end{abstract}

Objective: To investigate how lgG trafficking from cerebrospinal fluid to brain parenchyma can be influenced by injury. Methods: IgG from healthy donors was intrathecally injected into the cerebrospinal fluid via cisterna magna at 1, 2, 4, or 7 days after a distal stereotactic sterile needle insertion to the striatum.

Results: Antibody deposition, detected by staining for human lgG, peaked 1 day after the intrathecal injection and was selectively seen close to the needle insertion. When NMO-lgG was intrathecally injected, we observed complementdependent NMO-like pathology (loss of aquaporin-4 and glial fibrillary acidic protein) proximal to the insertion site, with similar kinetics. A fluorescent tracer did not show the same distribution indicating lgG-selective localization.

Conclusion: These findings suggest that lgG from cerebrospinal fluid localize selectively in brain parenchyma at the site of injury and pathogenic NMO-IgG induce astrocyte pathology at the same location.

Keywords: Immunoglobulin-G, Cerebrospinal fluid, Neuromyelitis optica spectrum disorders, Pathology, Deposition

\section{Introduction}

Inflammatory demyelinating diseases of the central nervous system (CNS) result in destruction of the myelin sheath and nerve fibers, which results in disability [1]. Whereas multiple sclerosis (MS) is considered a disease with myelindirected immunity, in neuromyelitis optica spectrum disease (NMOSD), astrocytes are the primary target of the immune response $[1,2]$. In NMOSD, the target antigen is identified as aquaporin-4 (AQP4) [3]. In the CNS, AQP4 is expressed by astrocytes and localized to astrocytic end-feet at the blood-brain barrier (BBB) and ependymal cells at the cerebrospinal fluid (CSF)-brain barrier [4, 5]. NMOSD lesions are predominantly located at the optic nerve or spinal cord, but specific brain MRI abnormalities including

\footnotetext{
* Correspondence: towens@health.sdu.dk

${ }^{1}$ Neurobiology, Institute of Molecular Medicine, University of Southern

Denmark, JB. Winsloewsvej 25, 5000 Odense, Denmark

Full list of author information is available at the end of the article
}

brainstem lesions are being increasingly recognized $[6,7]$. Elevated titers of immunoglobulin-G (IgG) are seen in CSF in MS [8] and during relapse in NMOSD [9]. In NMOSD, anti-AQP4 antibodies in the CSF correlate with astrocyte damage, the primary pathologic process [10]. Deposition of IgG and complement is observed in NMOSD pathological lesions [11], as well as in a subset of MS patients with pattern II lesions [12]. There is a need for better understanding of the clinical relevance of antibodies in CSF and how they access the parenchyma.

Several animal models have been developed to examine the mechanism behind NMO-like pathology [13-16]. We have demonstrated that antibodies from NMO patients (NMO-IgG), with human complement that was intrathecally injected into the CSF via cisterna magna, induced NMO-like lesions with loss of AQP4 and glial fibrillary acidic protein (GFAP). These lesions co-localized with deposition of human IgG and activated complement. 
Notably, lesions were widespread in the brain parenchyma, and lesion location varied from mouse to mouse [15]. We showed that antibodies from the CSF were deposited via a paravascular route in subarachnoid and subpial space and in the periventricular region. Perivascular deposition was specific for NMO-IgG [14]. We further observed that NMO-IgG induced local complementdependent loss of BBB integrity at specific but variable sites within brain parenchyma [14].

There is broad consensus that AQP4/NMO-IgG initially enters the brain via BBB-deficient sites such as area postrema, where access to CSF occurs [17]. NMO-IgG from the CSF gains access to the brain parenchyma and induces NMO-like lesions [15], with BBB disruption, via a specific IgG distribution pattern [14]. There is a need for further studies of pathogenic mechanism of IgGs and a better comprehension of what influences distribution of CSF-derived IgG in the parenchyma, to help understand how this site specificity is determined. Whether and how fluid flow occurs from CSF to CNS parenchyma is currently under debate [18-20]. Because little is known about what determines CSF IgG distribution in parenchyma, we examined if external factors, such as a sterile needle insertion, could influence the distribution.

\section{Materials and methods}

\section{Animals}

Adult female $\mathrm{C} 57 \mathrm{Bl} / 6 \mathrm{~J}$ mice (Taconic, Lille Skensved, Denmark) were housed in The Biomedical Laboratory, University of Southern Denmark. Forty-six mice were included for examination of IgG or dextran distribution while four mice, one sample per brain hemisphere, were included for the gene expression analysis.

\section{Sterile needle insertion}

Mice were anesthetized by isoflurane inhalation. Stereotactic coordinates were $2 \mathrm{~mm}$ lateral from bregma and $0.2 \mathrm{~mm}$ anterior. A 30 -gauge needle attached to a $50-\mu \mathrm{l}$ Hamilton syringe was inserted $3.5 \mathrm{~mm}$ in the right hemisphere into the striatum. Ten microliters of PBS was infused $(2 \mu \mathrm{l} / \mathrm{min})$ following needle insertion. After injury, mice received Temgesic (RB Pharmaceuticals Limited, Berkshire, UK) for pain relief and isotonic $\mathrm{NaCl}$ subcutaneously to prevent dehydration.

\section{Intrathecal delivery}

One, 2, 4, or 7 days after the sterile needle insertion, a 30 -gauge needle (bent at $55^{\circ}, 2-2.5 \mathrm{~mm}$ from the tip) attached to a 50- $\mu$ l Hamilton syringe was inserted between the skull and the cervical vertebra into the intrathecal space of the cisterna magna. Three hundred micrograms of human antibodies either NMO-IgG or normal-IgG [15] and $288 \mu \mathrm{g}$ complement protein isolated from healthy donors or $125 \mu \mathrm{g}$ Dextran, fluoresceinated,
40,000 MW (Molecular Probes, Life Technologies, Eugene, OR, USA) in a total volume of $10 \mu \mathrm{l}$. Anesthesia and analgesia were as described above.

\section{Tissue processing}

Mice were euthanized by an overdose of sodium pentobarbital and subsequently perfused transcardially with ice-cold PBS (10 ml) followed by 4\% paraformaldehyde in PBS $(20 \mathrm{ml})$. After removal, brains were post-fixed in 4\% PFA, immersed in $30 \%$ sucrose in PBS for up to 2 weeks at $4{ }^{\circ} \mathrm{C}$, then frozen in cryostat embedding medium (Killik, Milano, Italy) by immersion in 2-methylbutane (Sigma-Aldrich, Denmark) in liquid nitrogen. For examination of dextran diffusion, whole cerebra were cut serially into $30-\mu \mathrm{m}$ coronal sections on a Micron HM 550 cryostat (Microm International $\mathrm{GmbH}$, Walldorf, Germany). For examination of IgG diffusion, $16-\mu \mathrm{m}$ sagittal or coronal serial sections through the lesion were cut on a Micron HM 550 cryostat. For examination of NMOSD-like pathology (loss of AQP4 and GFAP, deposition of IgG and C9neo, and BBB disruption), sagittal serial sections (50, 30, and $16 \mu \mathrm{m}$ ) were cut through the lesions. For examination of gene expression levels, mice were perfused with PBS, and a tissue block that included the needle insertion was stored in TRIzol reagent (Invitrogen-Molecular Probes, Eugene, OR, USA). For examination of $\mathrm{BBB}$ disruption, mice were intravenously injected with $2 \mathrm{mg}$ horseradish peroxide (HRP) $15 \mathrm{~min}$ before perfusion.

\section{Immunohistochemistry}

CNS tissue was evaluated for deposition of human IgG and C9neo, loss of AQP4, and GFAP staining.

Sections were dried then washed in PBS followed by three times wash in PBS containing 0.2\% Triton X-100 (Sigma-Aldrich, St. Louis, MO, USA) (PBST). Sections were then blocked for endogenous peroxidases by immersion in methanol containing $0.2 \% \mathrm{H}_{2} \mathrm{O}_{2}$ (Sigma-Aldrich, St. Louis, $\mathrm{MO}$, USA) for $30 \mathrm{~min}$ at room temperature. Sections were thereafter washed in PBST three times. Unless otherwise stated, washes were carried out three times and all incubations were at room temperature. After washing, sections were blocked for non-specific staining with 3\% bovine serum albumin (Sigma-Aldrich, St. Louis, MO, USA) in PBST for $30 \mathrm{~min}$. Sections were incubated for $1 \mathrm{~h}$ with primary antibody (rabbit anti-AQP-4 (1:400) (Alomone Labs Ltd., Jerusalem, Israel), rabbit anti-GFAP (1:1000) (DAKO Denmark A/S, Glostrup, Denmark), rabbit anti-human IgG (1:200) (Abcam, Cambridge, UK), rabbit anti-C9neo (1:100) (Abcam, Cambridge, UK)). To verify antibody specificity, sections were incubated with corresponding concentrations of rabbit immunoglobulin fraction (DAKO Denmark A/S, Glostrup, Denmark). Sections were then washed in PBST and incubated for $1 \mathrm{~h}$ with secondary antibody (biotinylated goat anti-rabbit IgG (1:2) (Abcam, Cambridge, UK)). After 
washing in PBST, sections were incubated for $1 \mathrm{~h}$ with streptavidin-horseradish peroxide (1:200, GE Healthcare, Little Chalfont, Buckinghamshire, UK). Afterwards, sections were washed in PBS and developed by adding 3,3'-diaminobenzidine (DAB, $0.5 \mathrm{mg} / \mathrm{ml}$ ) (Sigma-Aldrich, St. Louis, MO, USA) and $\mathrm{H}_{2} \mathrm{O}_{2}$ (0.033\%) (Sigma-Aldrich, St. Louis, MO, USA) for 2 min and washed twice in PBS. Finally, sections were dehydrated using increasing concentrations of ethanol, cleared in xylene, and mounted using DPX mounting medium (Merck KGaA, Darmstadt, Germany).

IgG deposition, BBB disruption, and loss of AQP4 staining were analyzed on full series (between 12 to 30 sections), while GFAP loss and deposition of C9neo were examined on corresponding sections. Images were acquired using an Olympus BX51 microscope with an Olympus DP73 camera (Olympus, Ballerup, Denmark) and analyzed using the free software Fiji.

\section{Immunofluorescence staining}

Dextran diffusion was investigated by co-staining for GFAP to identify the needle insertion. Slides with brain sections were dried and washed once in PBS and then three times in PBST. Sections were blocked for nonspecific staining as above then incubated for $1 \mathrm{~h}$ with anti-GFAP (1:1000, Cy3) (Sigma-Aldrich, St. Louis, MO, USA). Slides were then washed once in PBS, then $5 \mathrm{~min}$ in PBS containing $300 \mathrm{nM}$ 4,6-diamidino-2-phenylindole (Invitrogen) to stain nuclei, then washed in PBS and mounted using gelvatol [21].

Dextran diffusion was analyzed on a full series (approximately 40 sections). Images were acquired using an Olympus BX51 microscope with an Olympus DP73 camera and analyzed using Adobe Photoshop CS3 version 10.0.

\section{RNA extraction, reverse transcription, and quantitative reverse transcriptase/real-time PCR}

RNA was extracted using TRIzol reagent (Invitrogen-Molecular Probes, Eugene, OR, USA) in accordance with the manufacturer's protocol. One microgram of total RNA was reverse transcribed using M-MLV reverse transcriptase (Invitrogen) according to the manufacturer's protocol. Primer and probe sequences were as follows: angiotensinogen (AGT) (forward TGAACAACATTGGTGACACCAA, reverse CTGCTTTGAGTTCGAGGAGGAT, probe TGGGA GAGGTTCTCAATAG MGB), angiotensin-converting enzyme (ACE) (forward CCTCTGCCTGGGACTTCTACA, reverse CGTGACCCGTGTGCATTG, probe AAGGACTTCCGGATTAA MGB), renin (forward GCACCTTCAGTCTCCCAACAC, reverse CCCGGACAGAAGGCA TTTT, probe CTTTGAACGAATCCCGC MGB), IFN $\gamma$ (forward CATTGAAAGCCTAGAAAGTCTGAATAAC, reverse TGGCTCTGCAGGATTTTCATG, probe TCACCATCCTTTTGCCAGTTCCTCCAG MGB), interleukin (IL)-4 (forward ACAGGAGAAGGGACGCCAT, reverse
GAAGCCCTACAGACGAGCTCA, probe TCCTCACAGCAACGAAGAACACCACA MGB), IL-10 (forward GGTTGCCAAGCCTTATCGGA, reverse ACCTGCTC CACTGCCTTGCT, probe TGAGGCGCTGTCATCGAT TTCTCCC MGB), TGF $\beta$ (forward TGACGTCACTGGAGTTGTACGG, reverse GGTTCATGTCATGGATG GTGC, probe TTCAGCGCTCACTGCTCTTGTGACAG MGB), TNF $\alpha$ (forward CCAAATGGCCTCCCTCTCAT, reverse TCCTCCACTTGGTGGTTTGC, probe CTCACACTCAGATCAT MGB), and iNOS (gene expression assay kit from TaqMan, Applied Biosystems Inc., Foster City, CA, USA). Samples were run as triplicates on an ABI Prism 7300 Sequence Detection System (Applied Biosystems Inc.). Results were expressed relative to 18S rRNA $(2 \triangle \mathrm{CT}$ method) as endogenous control (TaqMan Ribosomal RNA control reagents kit; Applied Biosystems Inc). CDNA was diluted 1:1000 for 18S rRNA analysis.

\section{Statistics}

Data was analyzed by nonparametric Mann-Whitney $t$ test using GraphPad Prism version 4 (GraphPad Software Inc., San Diego, CA, USA). Data are presented as mean \pm SEM. Values of $p<0.05$ were considered statistically significant.

\section{Results}

Needle insertion into the brain parenchyma influences IgG trafficking

Groups of C57BL/6 mice received normal-human-IgG into the CSF by intrathecal injection $1,2,4$, or 7 days post stereotactic insertion of a sterile needle to striatum. Histopathological analysis showed significant deposition of human IgG in the ipsilateral hemisphere compared to the corresponding area in the contralateral and a lack of staining following intrathecal injection of PBS (Fig. 1a-f). IgG deposition was localized to the area immediately proximal to needle insertion. Controls for non-specific staining were negative (not shown). Immunohistochemical analysis revealed that IgG deposition in the area around the needle insertion was strongest at 1 day (Fig. 1a) and was gradually reduced at 2, 4, and 7 days after the needle insertion (Fig. 1b-d). Hematoxylin and eosin staining at the time point for the strongest IgG deposition revealed only few infiltrating cells in the area where IgG was deposited (not shown). Among inflammation-associated cytokines (IFN $\gamma$, IL-4, IL10, TGF $\beta$, TNF $\alpha$ ), only TNF $\alpha$ showed a significant upregulation by RT-qPCR (Fig. 1g).

\section{Distinct patterns of IgG versus dextran distribution}

To examine whether IgG simply followed CSF and interstitial fluid (ISF) flow, fluorescein-conjugated dextran $(40,000 \mathrm{MW})$ was injected intrathecally to mice. We observed a distribution pattern similar to that described by Iliff et al. [18] (not shown). We then examined the effect 


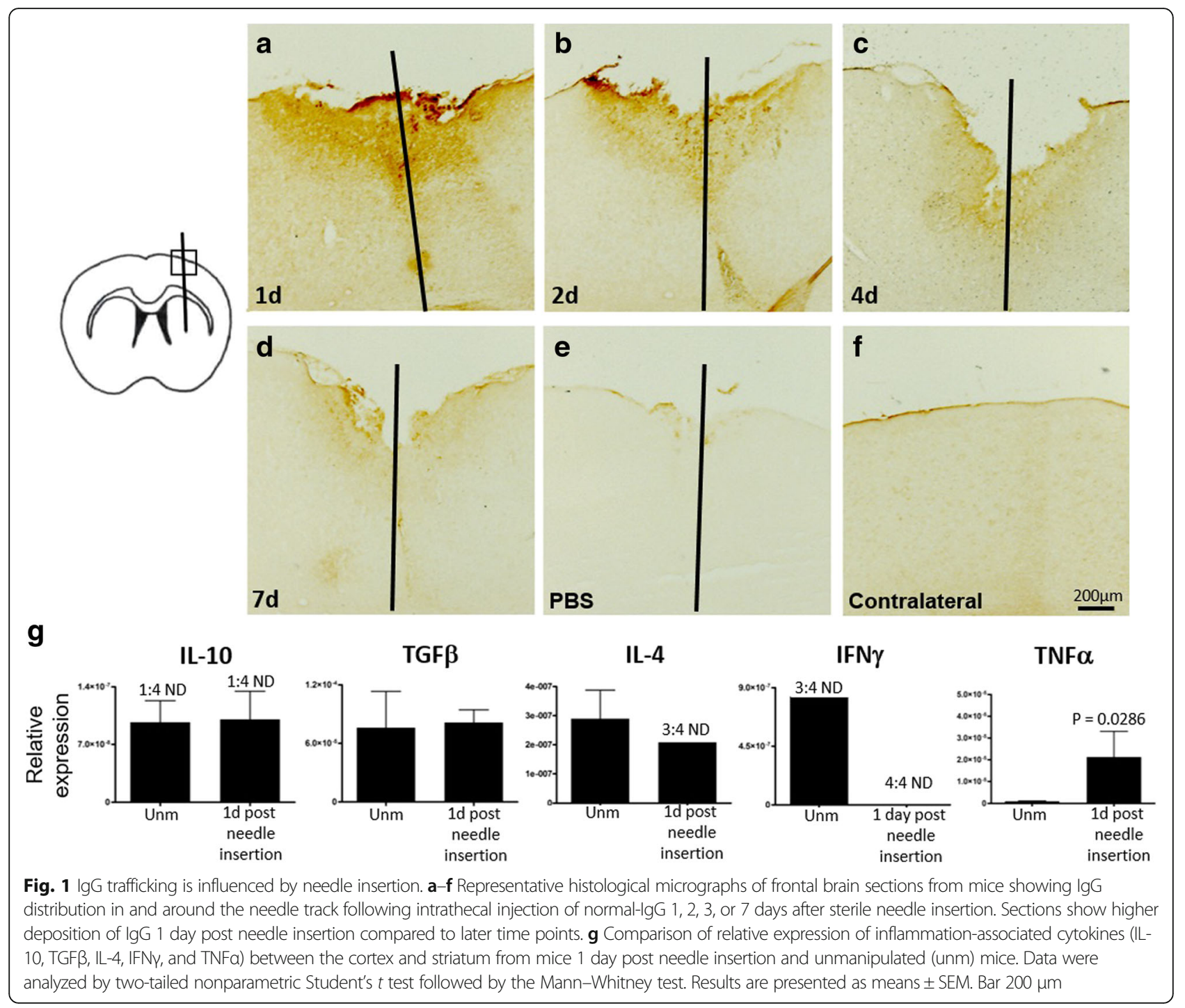

of a sterile needle insertion to the striatum. No change in distribution of dextran was observed when it was intrathecally injected into mice 1 day after sterile needle insertion (Fig. 2a). Importantly, we did not observe dextran deposition in the area around the needle insertion (Fig. 2c), although we observed increased GFAP staining at this site (Fig. 2c, white arrow). This indicates that altered CSF/ISF flow was not directly responsible for the pattern of IgG deposition seen following needle insertion. Analysis of expression levels of genes associated with the renin-angiotensin system and with inducible nitric oxide synthase 1 day post needle insertion also showed no change (not shown).

\section{Pathogenic IgG induces NMOSD-like pathology}

We asked whether intrathecally injected pathological human antibodies would result in NMOSD-like pathology at the insertion site. Mice received NMO-
IgG together with complement by intrathecal injection, or as controls NMO-IgG alone or normal-IgG with complement, 1 day post needle insertion. All groups showed significant deposition of human IgG at the insertion site in the ipsilateral hemisphere 1 day after the intrathecal injection (Fig. 3a). A low level of IgG deposition was detected at higher magnification throughout the areas adjacent to the interface with the CSF, possibly indicating passive diffusion of IgG from CSF to the parenchyma. This diffusion of IgG was strongest in mice that received NMO-IgG and complement, although was also detected in mice receiving NMO-IgG or normal-IgG + $\mathrm{C}$ (Fig. 3b). This low-level deposition did not result in noticeable loss of AQP4 or GFAP staining (not shown). Analysis of IgG deposition 3 days after intrathecal injection revealed that it was still significant at the insertion site for both NMO-IgG + 

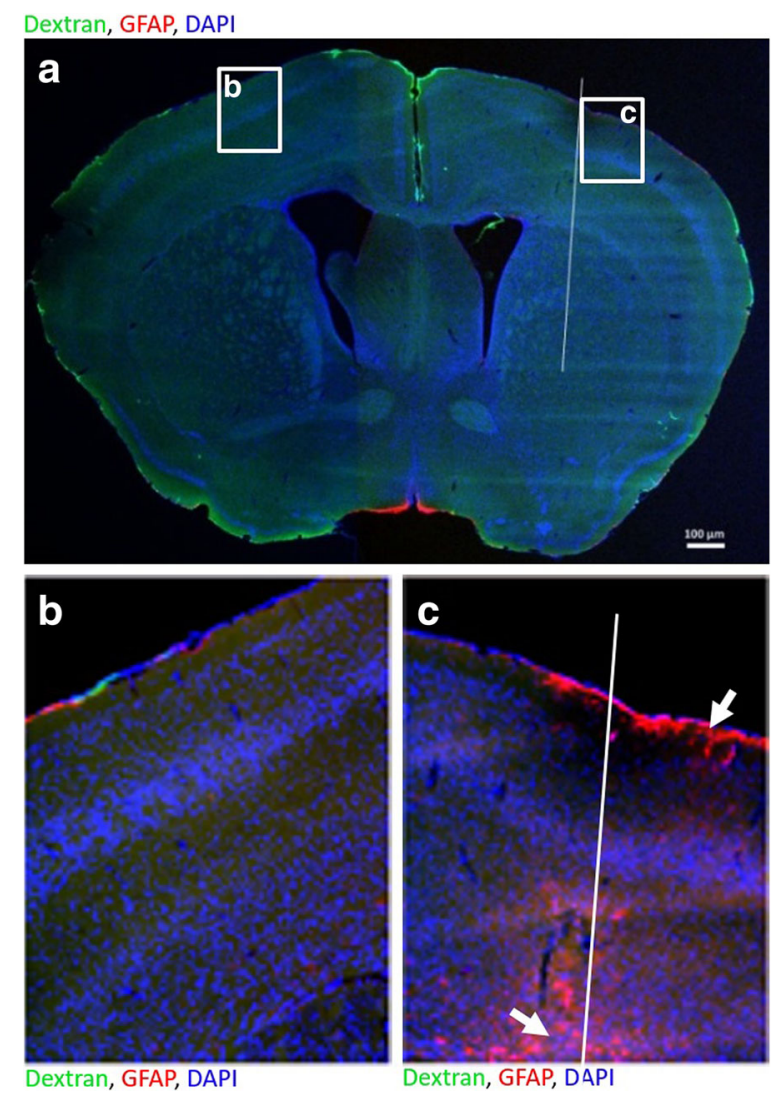

Fig. 2 Dextran does not deposit at needle insertion. a Representative frontal brain section of a mouse intrathecally injected with fluoresceinated dextran (40,000 MW) 1 day post sterile needle insertion. The section was co-stained anti-GFAP (red). Dextran was not observed in and around the needle track. $\mathbf{b}$ and $\mathbf{c}$ show higher magnification of the contralateral and ipsilateral hemispheres, respectively. GFAP staining (red) was used to identify the needle track (white arrow). Bar $100 \mu \mathrm{m}$

complement and for controls (Fig. 3c). However, the low-level IgG diffusion seen 1 day after intrathecal injection was now absent (Fig. 3d). Furthermore, 1 day post intrathecal injection complementdependent NMOSD-like pathology was observed at the site of sterile needle insertion in mice that received NMO-IgG and complement (3/3). Pathology was identified by loss of AQP4 and GFAP staining on astrocytes together with deposition of humanIgG and activated complement (C9neo) (Fig. 3e). One mouse that received NMO-IgG alone by intrathecal injection (1/4) showed loss of AQP4 and GFAP staining together with deposition of IgG and C9neo, suggesting unusual fixation of endogenous complement in this case (not shown). We also assessed BBB breakdown by intravenous injection of HRP. As expected, diffusion of HRP was observed associated to the needle track (not shown) and in association to NMO-like pathology (not shown).

\section{Discussion}

In this study, we have shown that independent of pathogenicity and complement, a sterile needle insertion directs deposition of CSF-derived IgG in the brain parenchyma. Time intervals between needle insertion and intrathecal injection of human IgG were shown to be crucial to the extent of IgG deposition, deposition peaking transiently 1 day after needle insertion. We observed complement-dependent astrocyte pathology at the needle insertion site induced by intrathecally delivered NMO-IgG and complement, identified as loss of staining for the astrocytic markers AQP4 and GFAP together with deposition of IgG and activated complement. Consistent with our previous study [14], we observed HRP leakage from blood to parenchyma in and close to the needle track and together with astrocytic pathology. Cellular infiltration was not marked. Importantly, intrathecally delivered dextran did not show the same localization. Expression levels of cytokines associated with inflammation were not affected by needle insertion, except for TNF $\alpha$ which was significantly upregulated. TNF $\alpha$ has previously been shown to be upregulated after injury in both adult and neonatal mouse brain [22].

Detection of AQP4-IgG in CSF has been reported in NMOSD, and disease activity has been linked to high levels of AQP4-IgG in CSF and serum [9, 10, 23]. Furthermore, levels of AQP4-IgG in CSF strongly correlate with astrocyte damage, as reflected in elevated levels of soluble GFAP in CSF during NMO relapse [10, 24]. We have previously reported that NMO-IgG and complement injected intrathecally deposited in three patterns. We observed deposition in leptomeninges, subarachnoid space, and the subpial spaces where vessels penetrate the brain parenchyma [14]. The previously observed leptomeningeal deposition of NMO-IgG is in line with the observation that contrast enhancement of the leptomeningeal structures, indicating disruption of the leptomeningeal blood-barrier, has been observed in AQP4-IgGpositive patients during clinical attacks [25]. The observed distribution pattern of IgG was similar for normal-IgG and NMO-IgG. We also observed deposition in the periventricular region for both normal- and NMO-IgG. Interestingly, we observed perivascular deposition specifically for NMO-IgG and complement at brain parenchymal vessels distal from the site of parenchymal entry of pial vessels. This perivascular deposition was associated with astrocyte pathology and together with blood-borne HRP leakage into the parenchyma indicated that NMO-IgG in CSF has potential widespread distribution within the brain via a paravascular route to exert pathologic effects [14]. In this study, we also observed HRP leakage together with astrocyte pathology. We propose that IgG intrathecally injected after needle insertion can distribute from CSF to parenchyma via 


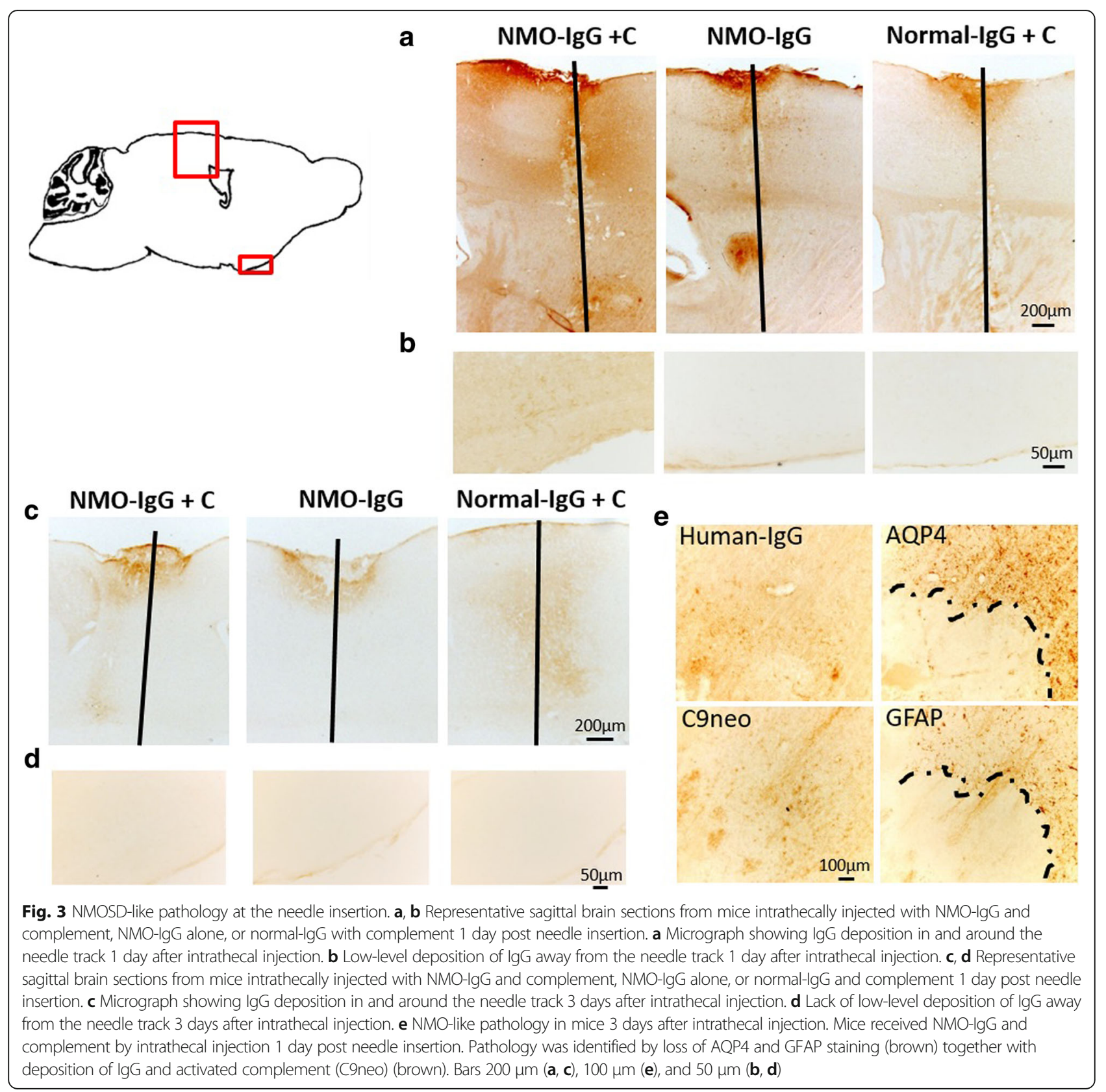

two different routes. One route would be via the above identified paravascular route, while the other route would be diffusion to parenchyma via the needle track.

It has been postulated that CSF flow is cardiac cycledependent and not unidirectional [19, 26, 27]. In principle, change in blood flow might affect the CSF flow, and a change in CSF flow could explain why antibodies deposited at the insertion site. However, examination of expression levels of iNOS and elements of the renin-angiotension system that are implicated in regulation of blood flow in the brain showed no change. Furthermore, intrathecal injection of a fluorescent dextran tracer showed no change in distribution between naïve mice and mice 1 day post needle insertion, indicating that CSF/ISF flow was not noticeably affected by the needle insertion. Increased AQP4 and GFAP immunoreactivities around the lesions were indeed observed, as also seen in clinical and experimental studies with or without needle insertion $[11,13]$. However, this traumainduced increased immunoreactivity of AQP4 cannot by itself explain the loss of AQP4 that we observed. NormalIgG, which does not include antibodies against AQP4, is also deposited around the needle insertion site, so upregulation of AQP4 cannot explain the IgG-specific deposition. The precise mechanism leading to deposition of IgG in and around the needle stick remains unclear. However, there appears to be selectivity for IgG deposition since a dextran 
tracer did not replicate these patterns. The mechanistic basis for this selectivity is unknown but may reflect Fc receptors or other Ig-binding structures in the CNS [28, 29].

This study confirms our previous finding that both NMO-IgG and normal-IgG diffuse from the CSF into the parenchyma after intrathecal injection via cisterna magna [14]. The low-level parenchymal IgG deposition bordering the CSF distal from the needle insertion that we observed after intrathecal injection is consistent with our previous study showing parenchymal IgG deposition 2 days post intrathecal injection [14]. Differences between the studies may reflect that whereas the localization of pathology and antibody distribution following intrathecal injection in our previous work [14] reflected three different deposition patterns, in the current study, a robustly predictable focus was created experimentally. Another study found that both NMO-IgG and normal-IgG were deposited in CNS parenchyma of rats when antibodies were continuously infused into the right ventricle using an osmotic mini pump [30]. In contrast, others found that when antibodies were infused into the intrathecal space in the spinal cord of rats and mice, only NMO-IgG infusion resulted in parenchymal deposition [31, 32].

Our observations suggest that IgG in CSF is distributed selectively from subarachnoid spaces to brain parenchyma at the site of an injury. We speculate that regional predilection of NMO lesions may reflect a combination of constitutive microenvironmental effects and external influences such as local infection which may also contribute to the localization of IgG deposition. The needle insertion that we have used is an experimental approach to model this hypothesis. Once the mechanism is established for IgG localization using experimental systems such as the one we describe, then the role of these different influences can be investigated.

\section{Abbreviations \\ AQP4: Aquaporin-4; BBB: Blood-brain barrier; CNS: Central nervous system CSF: Cerebrospinal fluid; GFAP: Glial fibrillary acidic protein; HRP: Horseradish peroxide; IFNy: Interferon gamma; IgG: Immunoglobulin-G; IL: Interleukin; ISF: Interstitial fluid; MS: Multiple sclerosis; NMO-lgG: Antibodies from NMO patients; NMOSD: Neuromyelitis optica spectrum disease; PBST: PBS containing 0.2\% Triton X-100; TGF $\beta$ : Transforming growth factor beta; TNFa: Tumor necrosis factor alpha}

\section{Acknowledgements}

We thank Dina S. Arengoth and Pia Nyborg Nielsen for advice on animal handling and operating procedures and technical support, respectively. We thank Søren Lillevang (Clinical Immunology Department, Odense University Hospital) and Lars Vitved (Department of Cancer and Inflammation Research, IMM, SDU) for antibody characterization and purification.

\section{Funding}

This research was supported by the Danish Multiple Sclerosis Society, Danish Agency for Science Technology and Innovation, The Lundbeck Foundation (\# R198-2015-171), Ph.D. stipend from The University of Southern Denmark Health Sciences Faculty, Bjarne Jensens Fond, Fonden for lægevidenskabelige fremme, Dagmar Marshalls Fond, and Familien Hede Nielsens Fond.

\section{Availability of data and materials}

All raw data in this manuscript are available on request.

\section{Authors' contributions}

MTM was responsible for stereotactic and intrathecal injections, daily monitoring of the mice, manuscript preparation, preforming histology, and statistical analysis. SFS was responsible for daily monitoring of the mice and preforming histology and RT-qPCR analysis. RK preformed stereotactic injection. TO, NA, and RK gave supervision on the design of the study and contributed significantly to the intellectual development of the project and helped to draft the manuscript. All authors critically revised the manuscript and approved the final manuscript.

\section{Ethics approval and consent to participate}

Experiments were conducted in accordance with the National Ethical Committee, Animal Experiments Inspectorate under the Danish Ministry of Food, Agriculture and Fisheries, and The Danish Veterinary and Food Administration (approval number 2014-15-0201-00369). The use of human material was approved by the Committee on Biomedical Research Ethics for the Region of Southern Denmark (ref. no. S20080142).

\section{Competing interests}

The authors declare that they have no competing interests.

\section{Publisher's Note}

Springer Nature remains neutral with regard to jurisdictional claims in published maps and institutional affiliations.

\section{Author details}

${ }^{1}$ Neurobiology, Institute of Molecular Medicine, University of Southern Denmark, JB. Winsloewsvej 25, 5000 Odense, Denmark. ${ }^{2}$ Department of Neurology, Slagelse Hospital, Institute of Regional Health Research, Slagelse, Denmark.

Received: 13 February 2018 Accepted: 11 April 2018

Published online: 17 April 2018

\section{References}

1. Lassmann $\mathrm{H}$. Targeting intracerebral inflammation in multiple sclerosis: is it feasible? Acta Neuropathol. 2012;124:395-6.

2. Hinson SR, Lennon VA, Pittock SJ. Autoimmune AQP4 channelopathies and neuromyelitis optica spectrum disorders. Handb Clin Neurol. 2016;133:377-403.

3. Zekeridou A, Lennon VA. Aquaporin-4 autoimmunity. Neurol Neuroimmunol Neuroinflamm. 2015;2:e110.

4. Verkman AS, Anderson MO, Papadopoulos MC. Aquaporins: important but elusive drug targets. Nat Rev Drug Discov. 2014;13:259-77.

5. Amiry-Moghaddam M, Lindland H, Zelenin S, Roberg BA, Gundersen BB, Petersen P, Rinvik E, Torgner IA, Ottersen OP. Brain mitochondria contain aquaporin water channels: evidence for the expression of a short AQP9 isoform in the inner mitochondrial membrane. FASEB J. 2005;19:1459-67.

6. Kremer L, Mealy M, Jacob A, Nakashima I, Cabre P, Bigi S, Paul F, Jarius S, Aktas $\mathrm{O}$, Elsone $\mathrm{L}$, et al. Brainstem manifestations in neuromyelitis optica: a multicenter study of 258 patients. Mult Scler. 2014;20:843-7.

7. Kim HJ, Paul F, Lana-Peixoto MA, Tenembaum S, Asgari N, Palace J, Klawiter EC, Sato DK, de Seze J, Wuerfel J, et al. MRI characteristics of neuromyelitis optica spectrum disorder: an international update. Neurology. 2015;84:1165-73

8. Compston A, Coles A. Multiple sclerosis. Lancet. 2008:372:1502-17.

9. Jarius S, Franciotta D, Paul F, Ruprecht K, Bergamaschi R, Rommer PS, Reuss R, Probst C, Kristoferitsch W, Wandinger KP, Wildemann B. Cerebrospinal fluid antibodies to aquaporin-4 in neuromyelitis optica and related disorders: frequency, origin, and diagnostic relevance. J Neuroinflammation. 2010:7:52.

10. Sato DK, Callegaro D, de Haidar Jorge FM, Nakashima I, Nishiyama S, Takahashi T, Simm RF, Apostolos-Pereira SL, Misu T, Steinman L, et al. Cerebrospinal fluid aquaporin-4 antibody levels in neuromyelitis optica attacks. Ann Neurol. 2014;76:305-9.

11. Lucchinetti CF, Mandler RN, McGavern D, Bruck W, Gleich G, Ransohoff RM, Trebst C, Weinshenker B, Wingerchuk D, Parisi JE, Lassmann H. A role for humoral mechanisms in the pathogenesis of Devic's neuromyelitis optica. Brain. 2002;125:1450-61. 
12. Jarius S, Konig FB, Metz I, Ruprecht K, Paul F, Bruck W, Wildemann B. Pattern II and pattern III MS are entities distinct from pattern I MS: evidence from cerebrospinal fluid analysis. J Neuroinflammation. 2017;14:171.

13. Bradl $\mathrm{M}$, Lassmann $\mathrm{H}$. Experimental models of neuromyelitis optica. Brain Pathol. 2014;24:74-82.

14. Asgari N, Berg CT, Morch MT, Khorooshi R, Owens T. Cerebrospinal fluid aquaporin-4-immunoglobulin G disrupts blood brain barrier. Ann Clin Transl Neurol. 2015;2:857-63.

15. Asgari N, Khorooshi R, Lillevang ST, Owens T. Complement-dependent pathogenicity of brain-specific antibodies in cerebrospinal fluid. J Neuroimmunol. 2013;254:76-82

16. Saadoun $S$, Waters $P$, Bell BA, Vincent A, Verkman AS, Papadopoulos MC Intra-cerebral injection of neuromyelitis optica immunoglobulin $\mathrm{G}$ and human complement produces neuromyelitis optica lesions in mice. Brain. 2010;133:349-61.

17. Asgari N, Skejoe HP, Lennon VA. Evolution of longitudinally extensive transverse myelitis in an aquaporin-4 lgG-positive patient. Neurology. 2013;81:95-6.

18. Iliff JJ, Wang M, Zeppenfeld DM, Venkataraman A, Plog BA, Liao Y, Deane R, Nedergaard M. Cerebral arterial pulsation drives paravascular CSF-interstitial fluid exchange in the murine brain. J Neurosci. 2013;33:18190-9.

19. Bulat $M$, Klarica $M$. Recent insights into a new hydrodynamics of the cerebrospinal fluid. Brain Res Rev. 2011;65:99-112.

20. Abbott NJ, Pizzo ME, Preston JE, Janigro D, Thorne RG. The role of brain barriers in fluid movement in the CNS: is there a 'glymphatic' system? Acta Neuropathol. 2018;135:387-407.

21. Khorooshi R, Owens T. Detection and cellular localization of phospho-STAT2 in the central nervous system by immunohistochemical staining. Methods Mol Biol. 2013;967:179-88.

22. Rostworowski M, Balasingam V, Chabot S, Owens T, Yong WW. Astrogliosis in the neonatal and adult murine brain post-trauma: elevation of inflammatory cytokines and the lack of requirement for endogenous interferon-gamma. J Neurosci. 1997;17:3664-74.

23. Takahashi T, Fujihara K, Nakashima I, Misu T, Miyazawa I, Nakamura M, Watanabe S, Shiga Y, Kanaoka C, Fujimori J, et al. Anti-aquaporin-4 antibody is involved in the pathogenesis of NMO: a study on antibody titre. Brain 2007;130:1235-43.

24. Takano R, Misu T, Takahashi T, Sato S, Fujihara K, Itoyama Y. Astrocytic damage is far more severe than demyelination in NMO: a clinical CSF biomarker study. Neurology. 2010;75:208-16.

25. Asgari N, Flanagan EP, Fujihara K, Kim HJ, Skejoe HP, Wuerfel J, Kuroda H, Kim SH, Maillart E, Marignier R, et al. Disruption of the leptomeningeal blood barrier in neuromyelitis optica spectrum disorder. Neurol Neuroimmunol Neuroinflamm. 2017;4:e343.

26. Oreskovic $D$, Klarica $M$. The formation of cerebrospinal fluid: nearly a hundred years of interpretations and misinterpretations. Brain Res Rev. 2010; 64:241-62.

27. Bulat M, Lupret V, Orehkovic D, Klarica M. Transventricular and transpial absorption of cerebrospinal fluid into cerebral microvessels. Coll Antropol. 2008:32(Suppl 1):43-50

28. Lunnon K, Teeling JL, Tutt AL, Cragg MS, Glennie MJ, Perry VH. Systemic inflammation modulates Fc receptor expression on microglia during chronic neurodegeneration. J Immunol. 2011;186:7215-24.

29. Teeling JL, Carare RO, Glennie MJ, Perry VH. Intracerebral immune complex formation induces inflammation in the brain that depends on Fc receptor interaction. Acta Neuropathol. 2012;124:479-90.

30. Marignier R, Ruiz A, Cavagna S, Nicole A, Watrin C, Touret M, Parrot S, Malleret G, Peyron C, Benetollo C, et al. Neuromyelitis optica study model based on chronic infusion of autoantibodies in rat cerebrospinal fluid. J Neuroinflammation. 2016;13:111.

31. Geis C, Ritter C, Ruschil C, Weishaupt A, Grunewald B, Stoll G, Holmoy T, Misu T, Fujihara K, Hemmer B, et al. The intrinsic pathogenic role of autoantibodies to aquaporin 4 mediating spinal cord disease in a rat passive-transfer model. Exp Neurol. 2015;265:8-21.

32. Zhang H, Verkman AS. Longitudinally extensive NMO spinal cord pathology produced by passive transfer of NMO-lgG in mice lacking complement inhibitor CD59. J Autoimmun. 2014:53:67-77.

\section{Ready to submit your research? Choose BMC and benefit from:}

- fast, convenient online submission

- thorough peer review by experienced researchers in your field

- rapid publication on acceptance

- support for research data, including large and complex data types

- gold Open Access which fosters wider collaboration and increased citations

- maximum visibility for your research: over $100 \mathrm{M}$ website views per year

At BMC, research is always in progress.

Learn more biomedcentral.com/submissions 